\title{
Effects of noise and working memory capacity on memory processing of speech for hearing-aid users
}

Hoi Ning, Elaine Ng, Mary Rudner, Thomas Lunner, Michael Syskind Perdersen and Jerker

Rönnberg

\section{Linköping University Post Print}

\section{Tweet}

N.B.: When citing this work, cite the original article.

Original Publication:

Hoi Ning, Elaine Ng, Mary Rudner, Thomas Lunner, Michael Syskind Perdersen and Jerker Rönnberg, Effects of noise and working memory capacity on memory processing of speech for hearing-aid users, 2013, International Journal of Audiology, (52), 7, 433-441.

http://dx.doi.org/10.3109/14992027.2013.776181

Copyright: Informa Healthcare

http://informahealthcare.com/

Postprint available at: Linköping University Electronic Press

http://urn.kb.se/resolve?urn=urn:nbn:se:liu:diva-91780 
Effects of noise and working memory capacity on memory processing of speech for hearing aid users

$$
\begin{aligned}
& \text { Elaine Hoi Ning } \mathrm{Ng}^{1} \text {, Mary Rudner }{ }^{1} \text {, Thomas Lunner }{ }^{1,2,3} \text {, } \\
& \text { Michael Syskind Pedersen }{ }^{4} \text {, and Jerker Rönnberg }
\end{aligned}
$$

1 Linnaeus Centre HEAD, Swedish Institute for Disability Research, Department of Behavioural Sciences and Learning, Linköping University, Sweden

2 Eriksholm Research Centre, Oticon A/S, Snekkersten, Denmark

3 Department of Clinical and Experimental Medicine, Linköping University, Sweden

4 Oticon A/S, Smørum, Denmark

Keywords: Hearing aid, free recall, cognitive abilities, working memory, individual differences

$\begin{array}{ll}\text { Abbreviations: } \\ \text { 4T } & \text { Four-talker babble } \\ \text { ANCOVA } & \text { Analysis of Covariance } \\ \text { ANOVA } & \text { Analysis of Variance } \\ \text { HINT } & \text { Hearing In Noise Test } \\ \text { NoP } & \text { No processing } \\ \text { NR } & \text { Binary masking noise reduction } \\ \text { PTA } & \text { Pure-tone average } \\ \text { RS } & \text { Reading span } \\ \text { SNR } & \text { Signal-to-noise ratio } \\ \text { SSN } & \text { Steady-state noise } \\ \text { SWIR } & \text { Sentence-final Word Identification and Recall } \\ \text { TF } & \text { Time-frequency }\end{array}$

Correspondence author:

Elaine H. N. Ng

Department of Behavioral Sciences and Learning

Linköping University

SE-581 83 Linköping

Sweden

Tel: +46 13282218

Fax: +46 13282145

Email: hoi.ning.ng@liu.se 


\begin{abstract}
Objectives: It has been shown that noise reduction algorithms can reduce the negative effects of noise on memory processing in persons with normal hearing. The objective of the present study was to investigate whether a similar effect can be obtained for persons with hearing impairment and whether such an effect is dependent of individual differences in working memory capacity. Design: A sentence-final word identification and recall (SWIR) test was conducted in two noise backgrounds with and without noise reduction as well as in quiet. Working memory capacity was measured using a reading span (RS) test.

Study Sample: Twenty-six experienced hearing aid users with moderate to moderately severe sensorineural hearing loss.

Results: Noise impaired recall performance. Competing speech disrupted memory performance more than speech-shaped noise. For late-list items the disruptive effect of the competing speech background was virtually cancelled out by noise reduction for persons with high working memory capacity.

Conclusions: Noise reduction can reduce the adverse effect of noise on memory for speech for persons with good working memory capacity. We argue that the mechanism behind this is faster word identification that enhances encoding into working memory.
\end{abstract}


Introduction

Advanced signal processing in hearing aids is designed to enhance listening comfort and reduce listening effort, in addition to improving audibility and intelligibility. Listening effort may be proportional to the demand for cognitive processing resources. Sarampalis and colleagues (2009) showed that the Ephraim-Malah noise reduction algorithm (Ephraim \& Malah, 1985) reduced the effort of listening in noise for people with normal hearing and, what is more, improved their memory for heard materials. However, improved memory as a result of noise reduction for people with hearing impairment has not been reported. In this study, we investigated the effects of noise and binary masking noise reduction in hearing aids (Wang et al., 2009) on the ability of listeners with hearing impairment to recall speech heard in noise.

The presence of background noise, such as stationary noise and competing speech, makes speech understanding more effortful, especially for persons with hearing impairment (Rudner et al, 2011). For individuals with normal hearing, stationary noise may have a stronger masking effect than competing speech at the same signal-to-noise ratio (SNR) due to the absence of the temporal fluctuations found in speech which provide the opportunity to listen to the target speech in the dips in the background noise (Duquesnoy,1983). In other words, a competing speech background, which contains temporal fluctuations, may give rise to release from masking of target speech (Festen \& Plomp, 1990). However, the masking release in a competing speech background reduces as hearing loss increases and as signal-to-noise ratio (SNR) improves. Linguistic information in competing speech causes distraction and adds to speech understanding 
difficulties (Sörqvist \& Rönnberg, 2012). Therefore, for persons with hearing impairment, competing speech may have a stronger masking effect on speech than stationary noise.

Working memory, which involves simultaneous processing and storage of information, is limited, and its capacity varies across individuals (Daneman \& Carpenter, 1980). Depending on the difficulty of the listening conditions, the involvement of working memory in speech perception varies (Rudner et al., 2011). The Ease of Language Understanding (ELU) model describes the role that working memory capacity plays in speech perception in challenging listening conditions (Rönnberg, 2003; Rönnberg et al., 2008). In favorable circumstances, language input is intact and this gives better access to phonologically based long-term representations in the mental lexicon. Under such circumstances, processing of language input is automatic and implicit. When the input is weak or distorted, a mismatch may arise. In this situation, explicit processing is needed to match the suboptimal input with representations in the long-term memory store. Thus, efficient cognitive function and good cognitive capacity may support the effectiveness of remedial explicit processing.

Individual cognitive abilities and aided hearing

A number of studies have demonstrated the relationship between cognitive abilities and speech perception performance in hearing aid users. Lunner (2003) found that working memory capacity and phonological processing speed were positively correlated with aided speech perception performance. Similarly, Humes (2007) showed that once audibility has been controlled for, cognitive function is the most important predictor of speech understanding in 
noise in older adults. The role of individual cognitive differences has also been studied in aided speech perception in noise with various signal processing algorithms, such as compression settings. People with good cognitive abilities could benefit from fast release time of compression in modulated noise (Foo et al., 2007; Gatehouse et al., 2003; Moore, 2008); possibly due to better ability to listen in the gaps in noise. However, fast compression release may put people with below average cognitive abilities at a disadvantage (See e.g. Foo et al., 2007; Rudner et al., 2011).

Hearing aids with adequate amplification and appropriate signal processing (e.g. based on input from directional microphones) can make speech input audible, even in challenging or noisy listening conditions (Ricketts, 2005). In this way, less explicit processing may be required for speech perception and thus more resources may be made available for higher level processing of auditory inputs. However, signal processing may also introduce unwanted artifacts or distortions, which may lead to a need of engaging effortful explicit processing (Lunner et al., 2009). Hearing aid users with good working memory capacity gain more benefit from advanced signal processing probably because more resources are available to overcome the extra processing of the artificial or distorted signals (Rudner et al., 2011). Thus, whether or not a hearing aid user can take advantage of a specific signal processing algorithm including signal processing artifacts may depend on individual cognitive abilities.

Effects of noise on free recall performance

Memory for heard speech varies depending on age, hearing loss and the presence of interfering background noise, even when the materials are equally well perceived in different 
background conditions (McCoy et al., 2005; Rabbitt, 1990; Tun et al., 2002, 2009; Wingfield et al., 2005). Memory performance can be measured using a free recall test. In a study aimed at gauging the effects of noise reduction algorithms on cognitive resources during listening to speech in noise, Sarampalis et al. (2009) used a dual task paradigm, which involved repeating final words of sets of spoken sentences and encoding the final words into memory for subsequent recall (see Pichora-Fuller et al., 1995). They showed that noise impaired word recall in a competing speech background for young people with normal hearing, particularly for sentences at the beginning of the lists, and that this effect of noise was weakened when a noise reduction algorithm was applied. A large body of literature in the field of cognitive psychology demonstrates that whereas enhanced recall of early-list items (primacy effect) reflects improved encoding into long-term memory, enhanced recall of late-list items (recency effect) reflects improved encoding into working memory (Murdock, 1974). Thus, the results of Sarampalis et al. suggested that the presence of noise could disturb transfer of information contained in speech to long-term storage.

When speech stimuli are distorted, degraded by background noise or perceived by older listeners with hearing impairment, additional processing time may be required to achieve successful word identification (Heinrich \& Schneider, 2011; Tun et al., 2009). Heinrich and Schneider compared the ability to recall lists of words heard in quiet and in different types of interfering noise in young and older adults. Because the older adults had relatively limited resources, their recall performance was generally worse than that of the young adults. Among the older adults, who also had elevated high frequency hearing thresholds, better recall of words presented at the end of the lists in quiet than in various patterns of babble background was 
consistently observed. Better recall of words in early list positions in quiet than in various background patterns was also revealed, but in some conditions only. The authors argued that memory for items in late-list positions was related to the speed of encoding items into working memory. Perceiving speech in a competing speech background involves additional processing time, which may lead to slower and less efficient encoding. Hence, memory for late-list items would become vulnerable.

The present study

To investigate the effects of noise and noise reduction on memory for heard materials in people with hearing impairment, we used a test paradigm similar to that employed by Sarampalis et al. (2009). All sentence-in-noise stimuli were presented at the individualized SNR which predicted 95\% speech perception in stationary noise. Hence, the effect of noise on speech perception was kept at a minimum while potentially still taxing memory and elevating listening effort from the level experienced while listening to sentences in quiet.

The Ephraim-Malah noise reduction algorithm (Ephraim \& Malah, 1985) used by Sarampalis et al. (2009) is effective in attenuating constant (or stationary) noise. The binary masking algorithm, which is designed to maximize speech enhancement, is more effective when speech is masked by fluctuating noise or irrelevant speech (Brungart et al., 2006), and improves speech intelligibility in noise for individuals with normal hearing and hearing impairment (Wang et al., 2009). Clearer speech input may give a better representation in working memory and hence 
improve memory. Thus, we used binary masking, which is not currently commercially available, in the present study.

Two types of background noise, which are competing speech and stationary noise, were chosen in the study. The role of individual differences in cognitive ability on memory and use of signal processing was also examined.

Hypothesis 1: We predicted that noise would have an effect on memory in listeners with hearing impairment, such that memory performance for speech heard in noise would be worse than that in quiet.

Hypothesis 2: By introducing noise reduction, memory performance in noise would be at least partially restored.

Hypothesis 3: In addition, this effect on memory performance would be greater for items in late list positions because the participants in the current sample were older than those taking part in the study by Sarampalis et al (2009) and thus were likely to have slower memory encoding speeds (cf. Heinrich and Schneider, 2011; Tun et al., 2009).

Hypothesis 4: We expected that memory in competing speech would be worse than in stationary noise because of the stronger masking effect of competing speech.

Hypothesis 5: Since good cognitive abilities are associated with greater benefit from signal processing, we also predicted that overall memory performance would be better for persons with better working memory capacity when there was noise reduction and that this would be particularly apparent for vulnerable items in late list positions presented with a competing talker.

\section{Methods}


Participants

Twenty-six native Swedish speakers (15 women and 11 men) with symmetrical moderate to moderately-severe acquired sensorineural hearing loss were recruited from the audiology clinic of the University Hospital of Linköping, Sweden. Their average age was 59 years (SD = 7, range: 32-65 years) and their average pure-tone threshold (PTA) at .125, .25, .5, 1, 2, 3, 4, 6 and $8 \mathrm{kHz}$ in both ears was $49.77 \mathrm{~dB}$ HL (SD = 6.41). All were hearing aid users, with an average daily usage of 9.5 hours over 9 years, and they used digital hearing aids with common features such as wide dynamic range compression, noise reduction and directional microphones. To test whether the participants had age-appropriate cognitive performance, standardized tests of verbal information-processing speed, namely physical matching, lexical decision making and semantic decision making tests (cf. Rönnberg, 1990; Rönnberg et al., 1989) were administered. The test results (in response time) were within one standard deviation of the mean performance for older adults (mean age $=62$ years) reported by Rönnberg (1990). They had no history of otological problems or psychological disorders. The study was approved by the regional ethics committee and informed consent was obtained from all participants.

\section{Reading Span test}

The reading span (RS) test, which was originally developed by Daneman and Carpenter (1980), measures working memory capacity. The test used in this study was visually based and was a short version of the original Swedish RS test created by Rönnberg et al. (1989). A total of 24 instead of 54 sentences were presented. Lists of 3, 4, and 5 sentences were presented in ascending order of length, and two lists were presented at each list length. This test consisted of two parallel tasks. First, the participants had to judge whether three-word sentences shown on the 
center of a computer screen, at a rate of $800 \mathrm{msec}$ per word with an inter stimulus interval of 75 msec, were sensible or absurd (Baddeley et al., 1985). Then, after each list of sentences, the participants were prompted to recall either the first or the final words of the sentences in the list in correct serial order. They were encouraged to respond as accurately as possible. Two practice lists of 2 sentences were administered. The test was scored by the total number of items correctly recalled irrespective of serial order. This scoring procedure was used to optimize the individual variation in response and has been adopted in other studies (e.g. Lunner, 2003; Foo et al., 2007). The results of this test were used as a grouping criterion in investigating individual differences.

Sentence-final word identification and recall (SWIR) test

A subset of 140 Swedish Hearing In Noise Test (HINT) sentences (Hällgren et al., 2006) was used. These are everyday sentences without contextual constraints and the difficulty of the sentences has been equated. Each sentence was presented twice, yielding a total of 280 trials. According to Hällgren et al., the learning effect associated with hearing each sentence twice (i.e. short-term test-retest difference) was less than $1 \mathrm{~dB}$ in speech perception test. All the selected sentences ended in a bi- or tri-syllabic noun. Thirty-five lists of 8 sentences were formed. These lists were divided into two sets such that each sentence only occurred once in each set. In each list, six sentences ended with a bi-syllabic word and two with a tri-syllabic word. Word frequency of the sentence final words was calculated based on the Swedish PAROLE corpus, which contains 19 million words (Gellerstam et al., 2000). The average frequency was 560 and did not differ significantly between lists. 
The SWIR test consisted of two tasks which were performed in series. The participants were asked to report the final word of each sentence immediately after listening to it (identification task). They were encouraged to guess if they were unsure of the word. After reporting the final word of the eighth sentence of a list, they were asked to recall, in any order, all the words that they had previously reported (free recall task). Four practice sentence lists were administered.

\section{Test conditions of the SWIR test}

The sentences were presented in seven test conditions: Six noise backgrounds (three noise reduction conditions in two background noises) and in quiet as control.

Background noise. Two types of background noise, steady-state noise (SSN) and fourtalker babble (4T), were employed. The SSN was the stationary speech-shaped noise used in the Swedish HINT (Hällgren et al., 2006), which has the same long-term average spectrum as the HINT sentences. The 4T background, which is a competing speech background, consisted of recordings of two male and two female native Swedish speakers reading different paragraphs of a newspaper text. The 4T background was post-filtered to resemble the long-term average spectrum of the HINT sentences. Background noise was introduced 3 seconds prior to the onset of each sentence stimulus and was terminated 1 second after sentence offset. There was no noise when the final words were reported and recalled. Reverberation of about 350ms was also introduced in all conditions so that the auditory stimuli resembled real life listening situations. 
Noise reduction setting. In binary masking noise reduction mixtures of speech signal and noise are decomposed into segments called time-frequency (TF) units, by passing them through a 64-channel gammatone filterbank and then time-windowing the output of each filter. The local SNR of each TF unit is then compared to a preset SNR, which is known as the local criterion (LC). In order to make comparisons, target signals and background interference are estimated. When the local SNR of the TF unit is greater than the LC, which means the energy of the signal exceeds the energy of the noise, this TF unit is retained in the binary matrix. Otherwise, when the local SNR is less than the LC, the TF unit is reduced by $10 \mathrm{~dB}$. In this way, the TF units dominated by the interfering noise in the mixture are segregated from the target signal, and hence the resulting SNR of the processed mixtures becomes more favorable for speech perception even in adverse listening conditions (Brungart et al., 2006). The LC of $0 \mathrm{~dB}$ was chosen in this study in order to optimize the SNR gain with the binary masks (Li \& Wang, 2009).

There were three noise reduction settings in the present study: binary masking noise reduction (NR), an ideal version of NR (Ideal NR) and in absence of any noise reduction signal processing (NoP). The two binary masking conditions differed in terms of the availability of information input concerning the signal and noise in the mixture. In Ideal NR, complete information of both signal and noise was available before they were mixed to calculate the local SNR (see Wang et al., 2009 for details), while in NR, the local SNR was estimated during recording from the output of directional microphones pointing in opposite directions: from either the front (in the direction of target) or rear (in the direction of background talkers) (see Boldt et al., 2008 for details). Both algorithms gave $10 \mathrm{~dB}$ of noise reduction. Both Ideal NR and NR were used to determine whether these two variations of binary masking have differential effects 
on memory. The NoP condition was used as a baseline in order to determine the overall effect of noise reduction processing on memory.

Procedure

The data were collected at two sessions of approximately 2 hours each as part of a larger study. Audiometric measurements and the RS test were administered in the first session. In the second session, the SWIR test was performed. Prior to the administration of the SWIR test, an individualized SNR, which predicts 95\% speech perception in stationary noise, was obtained for each participant. There were two steps in estimating the individualized SNR: 1) SNR which yielded 84\% speech intelligibility in noise was estimated using the HINT procedure (see Hällgren et al., 2006) with a modified adaptive up-down procedure suggested by Levitt (1971). Each participant was required to listen to and repeat three original lists of Swedish HINT sentences (i.e. 30 sentences in total) in the standard Swedish HINT noise. None of these sentences were used in the SWIR test. Both speech and noise were first presented at $65 \mathrm{~dB}$ A (i.e. $0 \mathrm{~dB}$ SNR). The presentation level of noise varied according to the participant's response. The SNR decreased one step when 4 consecutive sentences were repeated correctly and increased one step whenever a sentence was not repeated correctly. The step size was $2 \mathrm{~dB}$ for the first 15 sentences and was then refined to $1 \mathrm{~dB}$ from the $16^{\text {th }}$ sentence onwards. 2) An individual psychometric function was plotted using the data points from the first step and the SNR predicting 95\% speech perception (mean $=4.11 \mathrm{~dB}$ SNR, SD = 1.85) was estimated from this function. This favorable SNR, which is higher than that of the speech threshold obtained at $50 \%$ or even $80 \%$ speech perception, was chosen to maximize speech perception in noise. The remaining resources could therefore be 
devoted to the recall task. This single individualized SNR was applied in all noise conditions; thus, SNR was held constant across conditions for each participant. This procedure was adopted to optimize the effect of condition. The drawback of applying the same SNR in all noise conditions is that actual intelligibility may differ across conditions. The presentation level of sentences was fixed at $65 \mathrm{~dB}$ A in all conditions.

The SWIR test was administered after obtaining the individualized SNR. All auditory stimuli were preprocessed using Matlab, generated with a high-quality 24-bit external PC soundcard (ECHO Audiofire 8) at a sampling rate of $22050 \mathrm{~Hz}$, and transmitted to the microphone of an Oticon Epoq XW behind-the-ear hearing aid in an anechoic chamber (Brüel \& Kjær, type 4232) through a measuring amplifier (Brüel \& Kjær, type 2636). In order to assure audibility, the hearing aid was further adjusted to give linear amplification according to each participant's hearing thresholds based on Voice Aligned Compression ${ }^{1}$, and the resulting program settings were then modified using special programming software to provide a linear 1:1 compression ratio for pure-tone input levels in the range from $30 \mathrm{~dB}$ to $90 \mathrm{~dB}$ SPL. This ensured that the level distributions of all signals and noises used in the subsequent measurements were within the region of linear compression ratio and thus unaffected by any compression kneepoint or output limiting. The receiver of the hearing aid was coupled with an IEC-711 ear simulator (Brüel \& Kjær, type 4157) and the auditory stimuli were then transmitted through an equalizer (Behringer, Ultra-Curve Pro Model DEQ2496) and another measuring amplifier (Brüel \& Kjær, type 2636), to a pair of ER3A insert earphones, which was routed into a double-walled sound booth where the participant was sitting. 
In the SWIR test, the orders of presentation of sentences within lists, lists within each set, and test conditions were randomized. Each participant was tested with five sentence lists in each test condition. All lists were presented once only but in all seven test conditions across participants in a counterbalanced manner.

Scoring method

The identification and free recall tasks in the SWIR test were scored as the percentage of responses given and correct recall of these responses (i.e. an incorrectly identified word could also be scored as a correct recall) per list respectively. Further, the list position of the recalled words was also analyzed. The $1^{\text {st }}$ to $3^{\text {rd }}, 4^{\text {th }}$ to $6^{\text {th }}$, and $7^{\text {th }}$ and $8^{\text {th }}$ items in each sentence list were partitioned into the primacy, asymptote, and recency positions correspondingly.

Although the participants were encouraged to give a response after listening to each sentence, perceptual errors were still expected occasionally because the presentation level was estimating speech intelligibility below $100 \%$. Therefore, whenever a response was not given in the identification task, the percentage of correct recall was calculated based on the total number of responses given instead of the total number of items in a list. This conservative method was used to make a fair comparison of serial recall in different test conditions (cf. Murphy et al., 2000).

Results 
In this section, the results of the RS test, which is also involved in the analyses of the SWIR test, are first reported. Prior to the analyses of the SWIR test, performance with the noise reduction settings NR and Ideal NR were compared. For the free recall part of SWIR, the learning effect and the relationship with RS performance were examined. To examine the interaction between RS and memory performance, two groups of participants were created based on the RS test scores (High and Low RS groups). Two different grouping methods were used and two sets of corresponding RS analyses were performed. In the first RS analysis, the two groups of participants were created using the median split technique ( $n=13$ in each group). In the second RS analysis, which supplements the first RS analysis, participants with mid-range performance on the RS test were excluded in creating the two groups ( $n=10$ in each group). To address the hypotheses concerning the effects of noise and noise reduction and effects of different types of background noise on memory, two Analyses of Variance (ANOVAs) were then carried out. RS was thus added as a between-subject factor in the ANOVAs.

\section{Reading Span test}

For the judgment task, the error rate was $7.5 \%$ which is considered to be low especially because the time given to the participants was rather brief and some of the sentences were somewhat ambiguous to judge. Thus, the performance of this task would not bias the interpretation of the results of this paper. An average of 10.36 items (SD = 3.38) was recalled out of 24 items. This result is comparable proportionally to that reported in Lunner (2003) and Foo et al. (2007), where 54 items were used instead of 24. RS did not correlate with either age $(\mathrm{p}=.84)$ or PTA $(p=.60)$. 
Noise reduction settings

Performance with two of the three noise reduction settings (Ideal NR and NR) was compared for both the identification and free recall tasks of SWIR. For the identification task, the mean performance with Ideal NR and NR was $99.52 \%$ and $100 \%$ respectively in SSN and 99.62\% and 99.33\% respectively in 4T. For the free recall task, an ANOVA was performed on the two noise reduction settings (Ideal NR, NR) and the two types of background noise (SSN, 4T). There was no main effect of noise reduction setting, $\mathrm{F}(1,24)=.011, \mathrm{p}=.75$, and noise reduction setting neither interacted with $\mathrm{RS}, \mathrm{F}(1,24)=1.17, \mathrm{p}=.29$, nor with RS and background noise together, $F(1,24)=.47, p=.50$. Since the effect of the two noise reduction settings on memory did not differ statistically and the pattern of performance for Ideal NR was indistinguishable from that for NR, one of the noise reduction setting conditions was removed from further analysis so that the variance in the ANOVA was reduced, making the analysis more sensitive to predicted interactions between noise reduction and serial position. The NR condition was included because it is a practically realizable version of Ideal NR, which is based on estimations of binary masks rather than idealized a priori binary masks from Ideal NR, and it can be implemented in hearing aids. From this point on, the only noise reduction algorithm considered is NR.

\section{SWIR test}

For the identification task, the mean performance in quiet was $99.81 \%$, with NoP and NR were $99.52 \%$ and $100 \%$ in SSN, and $84.71 \%$ and $99.62 \%$ in 4 T respectively. Word identification reached $95 \%$ in all conditions except $4 \mathrm{~T} / \mathrm{NoP}$, which is probably due to application of the same SNR in all noise conditions. Nevertheless, the distribution of missing responses across serial positions was even, and fair comparison of recall performance across different conditions was 
possible by excluding missing responses in scoring the free recall task. The means and standard deviations of the free recall task are shown in Table 1.

Insert Table 1 about here

There was a learning effect associated with hearing each sentence twice in the free recall task. Mean scores were significantly higher at the second presentation, $\mathrm{F}(1,316)=-4.24, \mathrm{p}=.00$, but crucially, this effect did not interact with background condition, $F(4,621)=.48, p=.75$.

Correlations between RS and SWIR

The relationships between RS and recall performance in all test conditions were examined. RS significantly correlated with overall performance in quiet, SSN/NoP, SSN/NR and 4T/NR (correlation coefficients ranging from .47 to .58, ps $<.01$ ). The correlation coefficient between RS and performance in 4T/NoP was $.39(\mathrm{p}=.057)$. When the recall performance was analyzed as a function of list position, RS significantly correlated with recall performance in both primacy and asymptote positions in quiet $(r=.44$ and $.41, \mathrm{p}=.03$ and .04 correspondingly), the primacy position only in SSN/NoP, SSN/NR and 4T/NoP $(r=.44, .58$ and $.46, \mathrm{p}=.03, .00$ and.02 correspondingly) and the asymptote position only in 4T/NR $(r=.57, \mathrm{p}=.00)$. No significant correlation was found between RS and recall performance in the recency position in any background.

To further investigate the interactions between RS and memory performance, participants were divided into groups based on the RS test performance. Two sets of ANOVAs were 
performed. The first set of ANOVAs addressed hypotheses 1, 2, 3 and 5 while the second set of ANOVAs additionally addressed hypothesis 4 which could not be addressed by the first set of ANOVAs and extended the scope of hypothesis 5. Within each set of ANOVAs, two different RS analyses were performed. The first RS analysis was based on dividing the group on RS performance using a median split technique. This was done by splitting the entire group $(\mathrm{n}=26)$ into two at the median value of RS test scores. Similar median split analyses have been published elsewhere by our team (Rudner et al., 2011; Zekveld et al., 2012). The median test score was 10.5, and one group ( $\mathrm{n}=13$ ) with RS test scores less than or equal to 10 (Low RS group) and another group $(n=13)$ with scores greater than 10 (High RS group) were formed. The mean RS scores were $7.8(\mathrm{SD}=2.5)$ and $12.8(\mathrm{SD}=2.2)$ in the low and high RS groups respectively, and they were significantly different, $t(24)=-5.85, p=.00$. There was no significant difference in age, PTA or word identification performance (in any of the 5 test conditions) between the groups (t(24) $=-.27, \mathrm{p}=.79 ; \mathrm{t}(24)=-.63, \mathrm{p}=.54 ;$ and $\mathrm{t}(144)=.05, \mathrm{p}=.96$, respectively). Figure 1 shows the configuration of hearing loss for each of the participants in the high and the low RS groups.

Insert Figure 1 about here

In the second RS analysis, participants with mid-range RS scores were excluded, and only those with higher RS scores (range 12 to 16 , mean $=13.80, \mathrm{SD}=1.55, \mathrm{n}=10$ ) and lower RS scores (range 3 to 9 , mean $=7.00, \mathrm{SD}=2.40, \mathrm{n}=10$ ) were included. This second analysis was performed to check whether participants with mid-range results skewed the interactions. For both RS analyses, we report the significant interactions only, which proved to be informative. 
First set of ANOVAs (Hypotheses 1, 2, 3 and 5)

The first set of ANOVAs addresses the hypotheses that noise reduces memory performance in listeners with hearing impairment (hypothesis 1) and that this effect is modulated by NR in late-list positions (hypotheses 2 and 3) for persons with high working memory capacity (hypothesis 5). It was computed to compare recall performance in 4T with and without NR and in quiet. This ANOVA has two within-subject factors: noise (NoP, NR, quiet), position (primacy, asymptote, recency), and one between-subject factor: RS group (high, low). In the median split analyses (the first RS analysis), there were three significant main effects: noise, $F(2,48)=3.13$, p $=.05$, position, $\mathrm{F}(2,48)=75.98, \mathrm{p}=.00$, and RS group, $\mathrm{F}(1,24)=5.95, \mathrm{p}=.02$. When the main effect of noise was examined, it was found that the recall performance in quiet was better than that in $4 \mathrm{~T} / \mathrm{NoP}, \mathrm{t}(144)=1.71, \mathrm{p}=.05$. Performance in $4 \mathrm{~T} / \mathrm{NR}$ was not significantly different from either that in quiet or in 4T/NoP. In other words, when there was noise reduction, the main effect of noise was no longer significant.

Investigation of the main effect of position showed that recall performance in the recency position was better than in the primacy position, $\mathrm{t}(144)=11.35, \mathrm{p}=.00$, which in turn was better than in the asymptote position, $\mathrm{t}(144)=3.55, \mathrm{p}=.00$. The high RS group performed better than the low RS group, which agrees with the correlation analyses results. Two two-way interactions were significant: noise $x$ RS and noise x position. The noise $x$ RS interaction, $F(2,24)=6.06$, $p$ $=.01$, showed that the high RS group performed better in $4 \mathrm{~T} / \mathrm{NR}, \mathrm{t}(144)=2.20, \mathrm{p}=.03$, and in quiet, $\mathrm{t}(144)=5.25, \mathrm{p}=.00$, than in $4 \mathrm{~T} / \mathrm{NoP}$, while there was no change of recall performance in the low RS group when noise was reduced or removed (Figure 2, left panel). The noise x position interaction, $\mathrm{F}(4,96)=3.48, \mathrm{p}=.01$, showed both $4 \mathrm{~T}$ conditions $(4 \mathrm{~T} / \mathrm{NR}$ and $4 \mathrm{~T} / \mathrm{NoP})$ compared 
to quiet affected recall negatively in the recency position, $\mathrm{t}(144)=2.27, \mathrm{p}=.02$ and $\mathrm{t}(144)=3.90$, $\mathrm{p}=.00$ respectively, but not in other positions (Figure 2, right panel). Further, the three-way interaction between noise, position and RS was significant, $\mathrm{F}(4,96)=2.42, \mathrm{p}=.05$.

The second RS analysis showed that this three-way interaction remained when the participants with mid-range RS were excluded from the analyses, $\mathrm{F}(4,72)=2.53, \mathrm{p}=.05$. Analysis of Covariance (ANCOVA) was also computed to investigate if the variance in memory performance was explained by age. When age was entered as a covariate into this ANOVA, the three-way interaction noise $\mathrm{x}$ position $\mathrm{x}$ RS remained significant, $\mathrm{F}(4,92)=2.51, \mathrm{p}=.05$.

Insert Figure 2 about here

The noise x RS interaction showed that the effect of noise on memory and its reduction by NR was attributable to the high RS group, in line with hypotheses 2 and 5 . The noise $x$ position interaction showed an effect in the recency position, also in line with hypothesis 3. Therefore, investigation of simple main effects in the three-way interaction was carried out on recall performance in the recency position for the high RS group only. Recall performance was significantly better in quiet than in $4 \mathrm{~T}$ with $\mathrm{NR}(\mathrm{t}(144)=4.26, \mathrm{p}=.01)$, which in turn was better than that in the 4T/NoP condition (Figure 3).

Insert Figure 3 about here

Second set of ANOVAs (Hypotheses 4 and 5) 
The hypothesis that competing speech would affect memory performance more than stationary noise for the hearing impaired participants in the present study (hypothesis 4) was examined by the second set of ANOVAs. This four-factor ANOVA had three within-subject factors: background type (SSN, 4T), noise reduction (NoP, NR) and position (primacy, asymptote, recency), and one between-group factor: RS group (high, low). In other words, the quiet condition was not included in testing the second hypothesis. In the median split analyses (the first RS analysis), the ANOVA showed a significant main effect of background type, where recall performance in SSN was better than in $4 \mathrm{~T}, \mathrm{~F}(1,24)=6.93, \mathrm{p}=.02$; a main effect of position, $\mathrm{F}(2$, $48)=72.10, \mathrm{p}=.00$; and a main effect of RS, $\mathrm{F}(1,24)=4.50, \mathrm{p}=.04$. However, there was no main effect of noise reduction, $\mathrm{F}(1,24)=.15, \mathrm{p}=.71$. $\mathrm{RS}$ also interacted with noise reduction, $\mathrm{F}(1,24)=6.51, \mathrm{p}=.02$ (Figure 4). The post hoc t-tests (Bonferroni adjusted for multiple comparisons at the .05 level) demonstrated that the high RS group outperformed the low RS group in the NR conditions, $\mathrm{t}(72)=4.24, \mathrm{p}=.00$, but not in the NoP conditions.

The second RS analysis showed that this interaction (RS x noise reduction) remained significant when the participants with mid-range RS were excluded, $F(1,18)=4.11, \mathrm{p}=.05$. When age was entered as a covariate into this ANOVA (i.e. ANCOVA), the two-way interaction RS $x$ noise reduction remained significant, $F(1,23)=6.49, \mathrm{p}=.02$. The four-way interaction was significant, $\mathrm{F}(2,48)=6.30, \mathrm{p}=.00$. To investigate the significant four-way interaction, two separate three-factor analyses of variance were performed, one in each of the background types, 4T and SSN. The factors included in both of these analyses were noise reduction, position and RS group. In $4 \mathrm{~T}$ the three-way interaction was significant, $\mathrm{F}(2,48)=5.37, \mathrm{p}=.01$, and showed 
results similar to those of the first set of ANOVAs. Therefore, further post-hoc analyses were not performed. In SSN, the simple three-way interaction was not significant, $F(2,48)=1.80, \mathrm{p}=.18$.

Insert Figure 4 about here

In summary, the correlation analysis showed a strong relationship between RS and recall performance in all test conditions except 4T/NoP. This relationship was further investigated using ANOVAs. The first set of ANOVAs showed a main effect of noise such that memory performance was poorer in $4 \mathrm{~T}$ than in quiet. However, the presence of NR reduced the adverse effect of noise on memory. In addition, a significant three-way interaction between noise, position and RS demonstrated that in the recency position in 4T for the high RS group NR significantly reduced the negative effect of noise on memory recall. The second set of ANOVAs demonstrated a main effect of background noise type demonstrating that $4 \mathrm{~T}$ disrupted memory performance more than SSN and an interaction between RS and noise reduction, such that recall performance in background conditions with NR was better in the high RS group than the low counterpart. These results were found to be robust when the differences in age were accounted for in the ANCOVAs.

\section{Discussion}

The results demonstrate that noise reduces recall of heard sentences in listeners with hearing impairment even when the speech materials are correctly perceived. This confirms hypothesis 1 and is in agreement with previous results (McCoy et al., 2005; Rabbitt, 1990; Tun et 
al., 2002, 2009; Wingfield et al., 2005). Interestingly, noise reduction reduces the adverse effect of noise on memory for persons with good working memory capacity. This confirms hypotheses 2 and 5. When words are perceived in the absence of noise, recall performance is comparatively better because less cognitive resources are required for efficient encoding (Murphy et al., 2000). Along the same lines, we argue that segregation of target speech from background is facilitated when noise is reduced. This means that the matching of lexical information of the target speech in long-term storage becomes less explicit and less cognitively demanding. Therefore, the adverse effect of noise on memory is reduced when there is noise reduction.

Effects of noise and individual differences on memory

Not surprisingly, the correlation analysis showed that participants with good working memory capacity performed better overall on the memory task than participants with poorer working memory. To further investigate the interaction effects of individual differences on memory performance, the data were analyzed by splitting the group by median RS. Although the split of the high and the low RS groups is entirely data-driven and the cut-off point used in this study should not be applied clinically, the analyses using this technique demonstrate that memory performance varies with noise and/or noise reduction in some but not all participants. For instance, there were significant effects of noise and noise reduction for individuals with high working memory only. These results help us to understand the interplay between different factors including individual differences, and how signal processing and background noise may have differential effects for hearing aid users. 
Unexpectedly, it was the participants with good working memory capacity whose memory performance was significantly disrupted by noise. As a corollary, it was only the high RS group whose memory performance in noise could be restored by noise reduction to a level similar to that found in quiet. Thus, we have shown that whereas noise has an effect on memory for heard materials in persons with hearing impairment, noise reduction can virtually cancel out this effect. This restorative effect of noise reduction is particularly salient in late-list positions for listeners with good working memory capacity. This finding of improved recall of words in late-list positions is in line with the results reported by Heinrich and Schneider (2011) for older participants. We extend the findings of Heinrich and Schneider by showing that the disruptive effects of noise on memory for late-list items can be counteracted for persons with hearing impairment by the use of noise reduction. It is possible that noise reduction allows speedier word identification such that encoding into working memory can consequently be speeded up and become more efficient, and hence recall performance is improved (Tun et al., 2009). This supports hypothesis 3.

A relationship between good cognitive abilities and the ability to make use of hearing aid signal processing for processing speech heard in noise has also been reported in previous studies. In the present study, a similar relationship between memory for speech perceived in noise and working memory capacity was established.

Noise did not reduce memory performance for listeners with low working memory capacity. People with limited cognitive abilities may not benefit from noise reduction signal processing algorithms in hearing aids (Rudner et al., 2011). This may be because any potential 
benefits provided by the signal processing are cancelled out by the extra cognitive demand exerted by the distortion attributable to signal processing artifacts (Lunner et al., 2009). The speech-in-noise mixture processed by the binary masking used in this study is highly intelligible, yet it does not sound as natural as unprocessed human speech in quiet because of the presence of artifacts generated during signal processing (Wang, 2008). This may introduce phonological mismatch between the incoming signal and the representation in long-term memory, which requires explicit processing and more cognitive resources (Rönnberg, 2003; Rönnberg et al., 2008; Lunner et al., 2009). In the present study, listeners with low capacity were neither affected by noise nor did they benefit from noise reduction. It is important to note that despite the potentially greater processing demand engendered by noise reduction for persons with lower working memory capacity, this group did not show poorer memory performance with noise reduction than in quiet.

Effects of background noise on memory

Better recall performance in stationary noise than in competing speech irrespective of signal processing was shown in the present study, which is in line with our prediction that competing speech has a stronger masking effect. This is in line with hypothesis 4 . Whenever there is background noise, obligatory processing of task-irrelevant sound is automatically engaged and this may lead to disruption of focal task (Hughes \& Jones, 2003). The changingstate nature of competing speech is known to be more disruptive than repetitive or steady-state sound in serial short-term memory (Jones \& Morris, 1992). 
Our results suggest that competing speech is more detrimental to recall performance than stationary noise. Listening in a competing speech background is more cognitively demanding than listening in stationary noise because lexical-semantic information in competing speech is more distracting (Sörqvist \& Rönnberg, 2012) and is harder to segregate from target speech (Mattys et al., 2009). Together with the poorer quality and audibility of sensory input at the acoustical and peripheral level, higher-order processing and attentional resources are therefore depleted in the presence of competing speech, which leads to a decline in memory (McCoy et al., 2005; Rabbitt, 1990; Tun et al., 2002, 2009; Wingfield et al., 2005).

Moreover, the executive/cognitive demand for inhibiting irrelevant linguistic information and resources for auditory stream segregation is generally higher in a competing speech background than in stationary noise (Macken et al., 2009). Further investigation should be focused on how individual differences in inhibition affect cognitive processing in hearing aid users.

\section{Conclusions}

In line with our predictions, the present study showed an effect of noise on memory for listeners with hearing-impairment, such that recall of heard speech was poorer with background noise than without and poorer in competing speech than in stationary noise. Interestingly, this effect of noise was restricted to persons with good working memory capacity. The effect of competing speech on memory was moderated by noise reduction, particularly in late-list positions for the same group of listeners. We argue that for persons with good working memory 
capacity, noise reduction allows speedier word identification and facilitates encoding of heard material into working memory.

\section{Acknowledgments}

The authors would like to thank Ulrik Kjems from Oticon A/S, Smørum, Denmark, for his contribution of ideas regarding the noise reduction signal processing, René Burmand Johannesson from the Oticon Research Centre Eriksholm, Denmark and Mathias Hällgren from the Department of Technical Audiology, Linköping University, Sweden, for their technical support, and Tomas Bjuvmar from the Hearing Clinic, University Hospital, Linköping, Sweden, for his assistance in data collection. 


\section{References}

Baddeley, A. D., Logie, R., \& Nimmo-Smith, I. (1985). Components of fluent reading. J Mem Lang, 24, 119-131.

Boldt, J. B., Kjems, U., Pedersen, M. S., et al. (2008, September). Estimation of the ideal binary mask using directional systems. Proceedings of the 11th International Workshop on Acoustic Echo and Noise Control.

Brungart, D. S., Chang, P. S., Simpson, B. D., et al. (2006). Isolating the energetic component of speech-on-speech masking with ideal time-frequency segregation. J Acoust Soc Am, 120, 4007-4018.

Daneman, M., \& Carpenter, P. A. (1980). Individual Differences in Working memory and Reading. J Verb Learn Verb Behav, 19, 450-466.

Duquesnoy, A. J. (1983) Effect of a single interfering noise or speech source upon the binaural sentence intelligibility of aged persons. J Acoust Soc Am, 74, 739-743.

Ephraim, Y., \& Malah, D. (1985). Speech enhancement using a minimum mean-square error logspectral amplitude estimator. IEEE Trans Acoust Speech Signal Processing, 33, 443-445.

Festen, J. M,. \& Plomp, R. (1990). Effects of fluctuating noise and interfering speech on the speech-reception threshold for impaired and normal hearing. J Acoust Soc Am, 88(4), 17251736.

Foo, C., Rudner, M., Rönnberg, J., et al. (2007). Recognition of speech in noise with new hearing instrument compression release settings requires explicit cognitive storage and processing capacity. J Am Acad Audiol, 18, 618-631.

Gatehouse, S., Naylor, G., \& Elberling, C. (2003). Benefits from hearing aids in relation to the interaction between the user and the environment. Int J Audiol, 42, S77-85. 
Gellerstam, M., Cederholm, Y., \& Rasmark, T. (2000). The bank of Swedish. Proceedings of the 2nd International Conference on Language Resources and Evaluation, Greece, 329-333.

Heinrich, A., \& Schneider, B. A. (2011). Elucidating the effects of ageing on remembering perceptually distorted word pairs. Q J Exp Psychol, 64, 186-205.

Hughes, R. W., \& Jones, D. M. (2003). Indispensable benefits and unavoidable costs of unattended sound for cognitive functioning. Noise Health, 6, 63-76.

Humes, L. E. (2007). The contributions of audibility and cognitive factors to the benefit provided by amplified speech to older adults. J Am Acad Audiol, 18, 590-603.

Hällgren, M., Larsby, B., \& Arlinger, S. (2006). A Swedish version of the Hearing In Noise Test (HINT) for measurement of speech Recognition. Int J Audiol, 45, 227-237.

Jones, D., \& Morris, M. (1992). Irrelevant speech and serial recall: implications for theories of attention and working memory. Scand J Psychol, 33, 212-229.

Levitt, H. (1971). Transformed Up-Down Methods in Psychoacoustics. J Acoust Soc Am, 49, 467-477.

Li, Y., \& Wang, D. (2009). On the optimality of ideal binary time-frequency masks. Speech Comm, 51, 230-239.

Lunner, T. (2003). Cognitive function in relation to hearing aid use. Int J Audiol, 42, S49- S58.

Lunner, T., Rudner, M., \& Rönnberg, J. (2009). Cognition and hearing aids. Scand J Psychol, 50, 395-403.

Macken, W. J., Phelps, F. G., \& Jones, D. M. (2009). What causes auditory distraction? Psychon Bul Rev, 16, 139-144.

Mattys, S. L., Brooks, J., \& Cooke, M. (2009). Recognizing speech under a processing load: dissociating energetic from informational factors. Cognit Psychol, 59, 203-243. 
McCoy, S.L., Tun, P. A, Cox, L. C., et al. (2005). Hearing loss and perceptual effort: downstream effects on older adults' memory for speech. Q J Exp Psychol A, 58(1), 22-33.

Moore, B. C. (2008). The choice of compression speed in hearing AIDS: theoretical and practical considerations and the role of individual differences. Trends Amplif, 12, 103-112.

Murdock, B. B. (1974). Human memory: Theory and data. Potomac, MD: Lawrence Erlbaum Associates.

Murphy, D. R., Craik, F. I., Li, K. Z., et al. (2000). Comparing the effects of aging and background noise on short-term memory performance. Psychol Aging, 15, 323-334.

Pichora-Fuller, M. K., Schneider, B. A., \& Daneman, M. (1995). How young and old adults listen to and remember speech in noise. J Acoust Soc Am, 97(1), 593-608.

Rabbitt, P. (1990). Mild hearing loss can cause apparent memory failures which increase with age and reduce with IQ. Acta Otolaryngol Suppl, 476, 167-175.

Ricketts, T. A. (2005). Directional hearing aids: then and now. J Rehabil R D, 42(4 Suppl 2), 133-144.

Rudner, M., Rönnberg, J., \& Lunner, T. (2011). Working memory supports listening in noise for persons with hearing impairment. J Am Acad Audiol, 22, 156-167.

Rönnberg, J. (1990). Cognitive and communicative function: the effects of chronological age and “handicap -age”. Eur J Cognit Psychol, 2, 253-274.

Rönnberg, J. (2003). Cognition in the hearing impaired and deaf as a bridge between signal and dialogue: a framework and a model. Int J Audiol, 42, s68-76.

Rönnberg, J., Arlinger, S., Lyxell, B., et al. (1989). Visual evoked potentials: relation to adult speechreading and cognitive function. J Speech Hear Res, 32, 725-735. 
Rönnberg, J., Rudner, M., Foo, C., et al. (2008). Cognition counts: A working memory system for ease of language understanding (ELU). Int J Audiol, 47, S99-105.

Sarampalis, A., Kalluri, S., Edwards, B., et al. (2009). Objective measures of listening effort: effects of background noise and noise reduction. J Speech Lang Hear Res, 52(5), 1230 1240.

Sörqvist, P., \& Rönnberg, J. (2012). Episodic long-term memory of spoken discourse masked by speech: What role for working memory capacity? J Speech Lang Hear Res,55 (1), 210-218.

Tun, P. A., McCoy, S., \& Wingfield, A. (2009). Aging, hearing acuity, and the attentional costs of effortful listening. Psychol Aging, 24(3), 761-766.

Tun, P. A., O'Kane, G., \& Wingfield, A. (2002). Distraction by competing speech in young and older adult listeners. Psychol Aging, 17(3), 453-467.

Wang, D. (2008). Time-frequency masking for speech separation and its potential for hearing aid design. Trends Amplif, 12, 332-353.

Wang, D., Kjems, U., Pedersen, M. S., et al. (2009). Speech intelligibility in background noise with ideal binary time-frequency masking. J Acoust Soc Am, 125, 2336-2347.

Wingfield, A., Tun, P. A., \& McCoy, S. L. (2005). Hearing loss in older adulthood: what it is and how it interacts with cognitive performance. Curr Dir Psychol Sci, 14, 144-148.

Zekveld, A. A., Rudner, M., Johnsrude, I. S., et al. (2012). Behavioral and fMRI evidence that cognitive ability modulates the effect of semantic context on speech intelligibility. Brain Lang, 122(2), 103-113. 


\section{Footnote}

${ }^{1}$ The Voice Aligned Compression (VAC) rationale can be classified as curvilinear widedynamic range compression that, compared to many other amplification strategies, provides less compression at high input levels and more compression at low input levels through lower compression kneepoints (varying between 30 and $40 \mathrm{~dB}$ SPL, depending on frequency region and amount of hearing loss).

This compression model is partly based on loudness data by Buus and Florentine (2001) and is intended to ensure improved sound quality without loss of speech intelligibility, rather than loudness compensation per se.

\section{Reference}

Buus, S., \& Florentine, M. (2001). Growth of loudness in listeners with cochlear hearing losses: Recruitment reconsidered. J Assoc Res Otolaryngol, 3, 120-139. 
Figure Captions
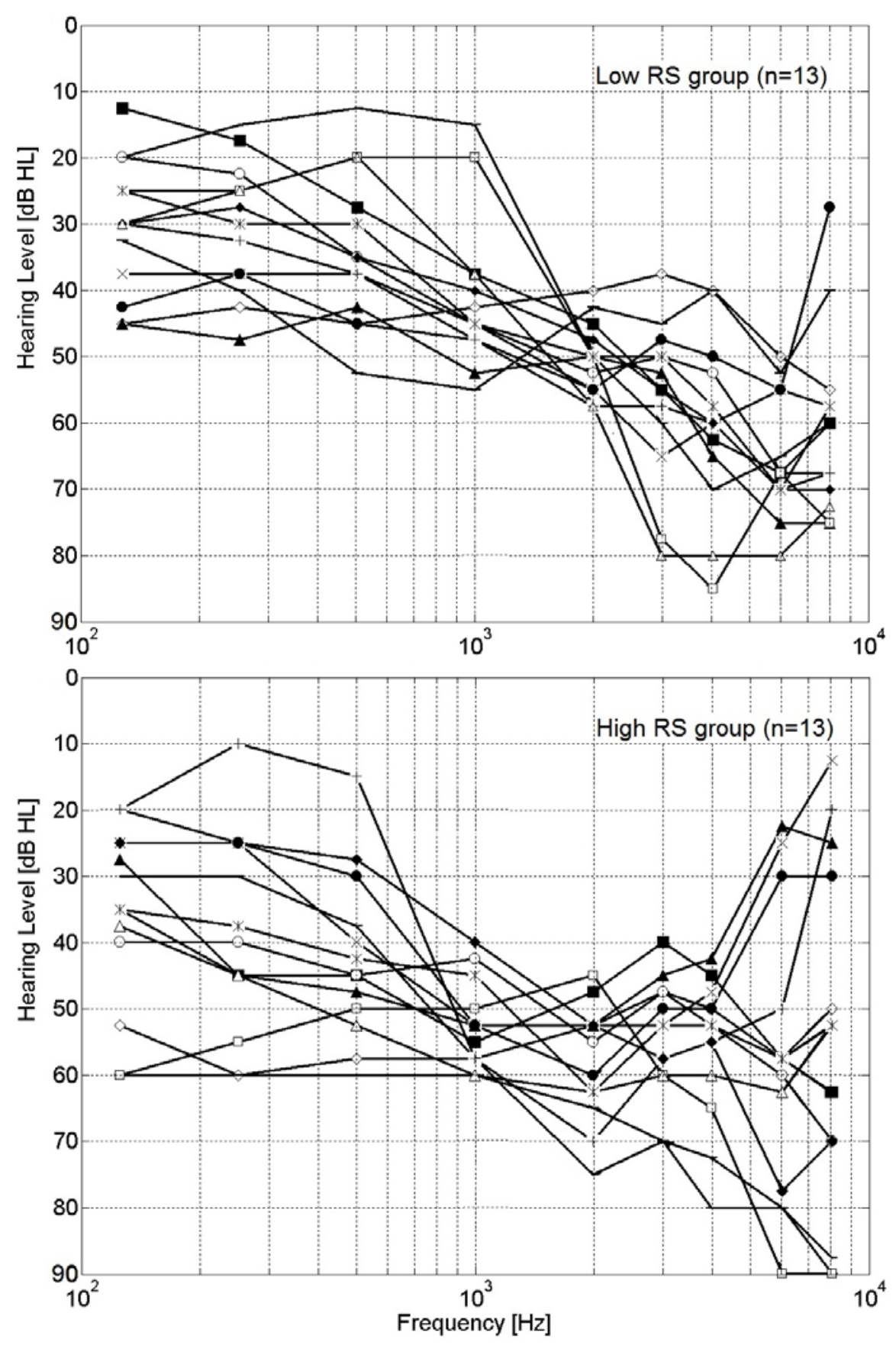

Figure 1. Configuration of hearing loss for each of the participants in the low (upper panel) and the high (lower panel) reading span (RS) groups. 

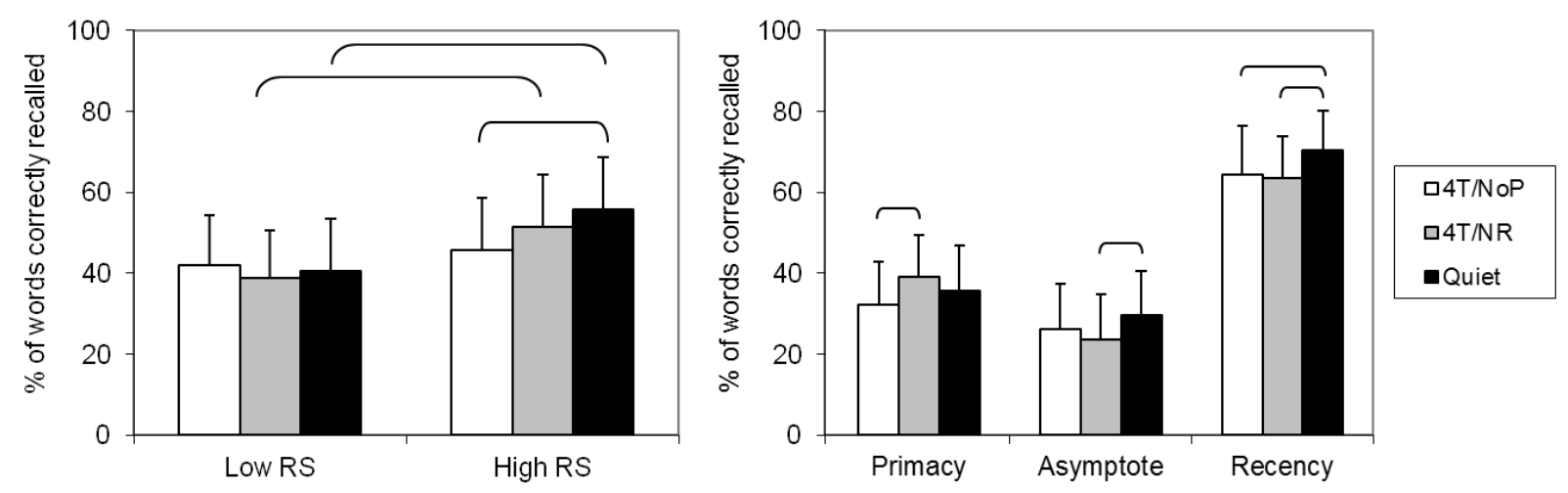

Figure 2. Significant two-way interactions between reading span (RS) and noise (left panel) and position and noise (right panel), including 4-talker babble without and with binary masking noise reduction (4T/NoP and 4T/NR respectively) and quiet. Error bars represent standard deviation.

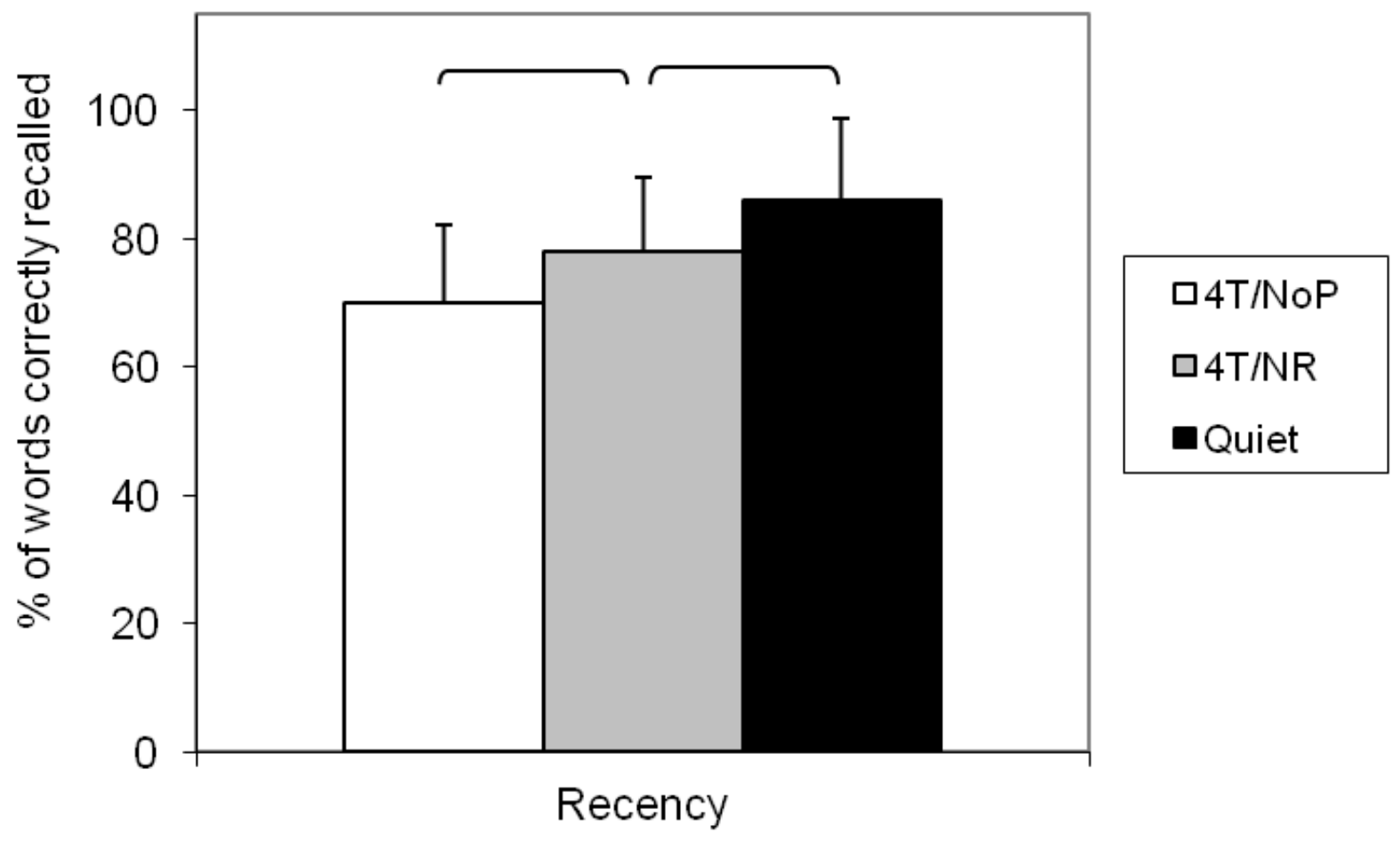

Figure 3. Investigation of simple main effects on recall performance in the recency position for the high reading span (RS) group only. Error bars represent standard deviation. 


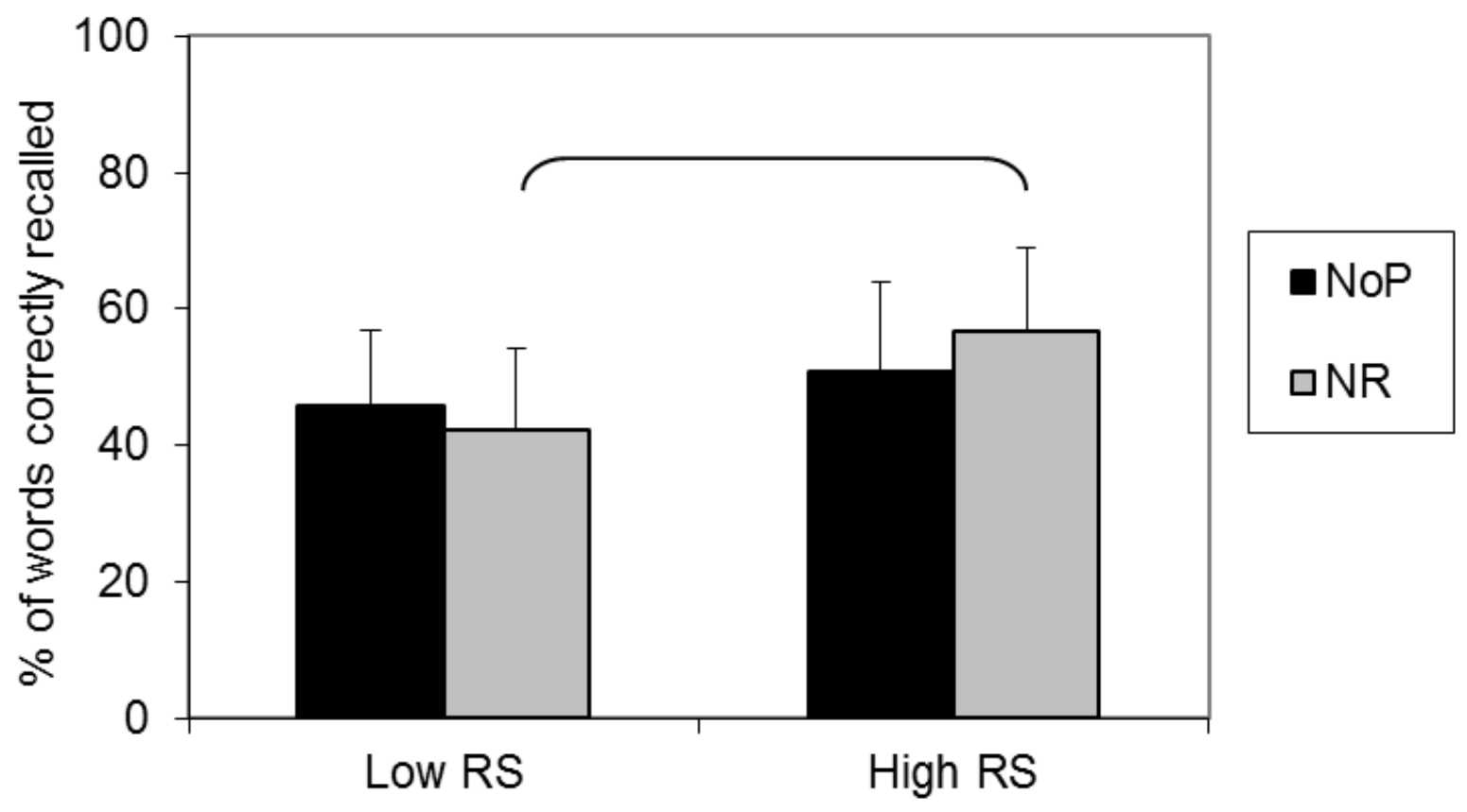

Figure 4. Significant two-way interaction between reading span (RS) and noise reduction: without (NoP) and with binary masking noise reduction (NR). Error bars represent standard deviation. 
Table 1. Means (in percentage) and standard deviations of the free recall task.

\begin{tabular}{|c|c|c|c|c|c|c|c|c|c|c|c|}
\hline \multirow{4}{*}{$\begin{array}{l}\text { Noise } \\
\text { reduction }\end{array}$} & \multirow{4}{*}{ RS } & & \multicolumn{6}{|c|}{ Background noise } & \multicolumn{3}{|c|}{ Quiet } \\
\hline & & & \multicolumn{3}{|c|}{ SSN } & \multicolumn{3}{|c|}{$4 \mathrm{~T}$} & & & \\
\hline & & & \multicolumn{3}{|c|}{ Serial position } & \multicolumn{3}{|c|}{ Serial position } & \multicolumn{3}{|c|}{ Serial position } \\
\hline & & & Primacy & Asymptote & Recency & Primacy & Asymptote & Recency & Primacy & Asymptote & Recency \\
\hline \multirow[t]{4}{*}{$\overline{\mathrm{NoP}}$} & High & $\mathbf{M}$ & 50.26 & 36.08 & 79.23 & 45.31 & 28.03 & 70.81 & 51.28 & 39.49 & 86.92 \\
\hline & & SD & 30.01 & 18.15 & 13.82 & 24.43 & 15.46 & 15.39 & 22.83 & 18.75 & 9.47 \\
\hline & $\overline{\text { Low }}$ & $\mathbf{M}$ & 37.95 & 24.71 & 79.23 & 28.96 & 31.82 & 77.55 & 29.74 & 27.77 & 75.38 \\
\hline & & SD & 26.72 & 12.18 & 11.15 & 23.72 & 12.14 & 15.03 & 23.35 & 17.33 & 14.50 \\
\hline \multirow[t]{4}{*}{$\overline{\mathrm{NR}}$} & High & $\mathbf{M}$ & 51.79 & 35.90 & 80.00 & 50.95 & 34.03 & 77.69 & & & \\
\hline & & SD & 20.03 & 21.52 & 14.14 & 20.12 & 15.98 & 12.35 & & & \\
\hline & $\overline{\text { Low }}$ & $\mathbf{M}$ & 26.15 & 27.18 & 73.08 & 39.74 & 19.49 & 66.67 & & & \\
\hline & & SD & 25.41 & 14.00 & 13.16 & 22.63 & 12.01 & 17.00 & & & \\
\hline
\end{tabular}

